Portland State University

PDXScholar

$7-12-2018$

\title{
An Indigenous Framework of the Cycle of Fetal Alcohol Spectrum Disorder Risk and Prevention Across the Generations: Historical Trauma, Harm and Healing
}

Kelly Gonzales

Portland State University, kelly.gonzales@pdx.edu

Michelle M. Jacob

University of Oregon

Amanda Mercier

Native Wellness Institute

Heather Heater

Future Generations Collaborative

Lindsay Nall Goes Behind

Euture Generations Collaborative Follow this and additional works at: https://pdxscholar.library.pdx.edu/sph_facpub

Part of the Public Health Commons

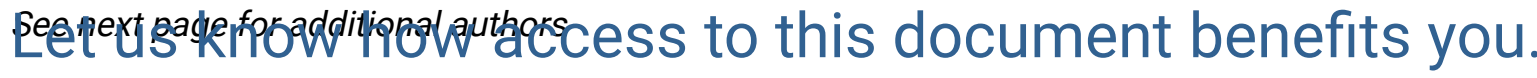

\section{Citation Details}

Gonzales, K. L., Jacob, M. M., Mercier, A., Heater, H., Nall Goes Behind, L., Joseph, J., \& Kuerschner, S. (2018). An indigenous framework of the cycle of fetal alcohol spectrum disorder risk and prevention across the generations: historical trauma, harm and healing. Ethnicity \& health, 1-19.

This Post-Print is brought to you for free and open access. It has been accepted for inclusion in OHSU-PSU School of Public Health Faculty Publications and Presentations by an authorized administrator of PDXScholar. Please contact us if we can make this document more accessible: pdxscholar@pdx.edu. 


\section{Authors}

Kelly Gonzales, Michelle M. Jacob, Amanda Mercier, Heather Heater, Lindsay Nall Goes Behind, Jillene Joseph, and Suzie Kuerschner 
Running Head: THE CYCLE OF TRAUMA AND FASD IN AI/AN COMMUNITIES

An Indigenous Framework of the Cycle of Fetal Alcohol Spectrum Disorder Risk and Prevention Across the Generations: Historical Trauma, Harm and Healing

Kelly L. Gonzales, MPH, PhD, corresponding author Assistant Professor Oregon Health Sciences University-Portland State University School of Public Health College of Urban \& Public Affairs

Portland State University

506 SW Mill Street, Suite 450

Portland, OR 97201

kelly.gonzales@pdx.edu

503-725-5108

Michelle M. Jacob, PhD

Associate Professor

Department of Education Studies

College of Education

5277 University of Oregon

Eugene, OR 97403

mjacob@uoregon.edu

Amanda Mercier, MS

Evaluator

Future Generations Collaborative

Native Wellness Institute

2830 SE Cleveland Drive

Gresham, OR 97080

anmpdx@gmail.com

Heather Heater, MPH

Program Manager

Future Generations Collaborative

Multnomah County Health Department

426 SW Stark Street

Portland, OR 97204

heather.heater@multco.us

Lindsay Nall Goes Behind, MSW

Health Policy Manager/Policy Coordinator

Future Generations Collaborative

Native American Youth and Family Services

5135 NE Columbia Blvd 
Portland, OR 97218

Jillene Joseph, BS

Executive Director

Native Wellness Institute

2830 SE Cleveland Drive

Gresham, OR 97080

jillene.joseph@frontier.com

Suzie Kuerschner

Executive Director

S.P.I.R.I.T.S

PO Box 27

Rhododendron, Oregon 97049

suziekuerschner@gmail.com

\section{Acknowledgments}

This work would not be possible without the dedication and courage of the Elders and Natural Helpers of the Future Generation Collaborative who served as research assistants. We are honored by the wisdom and experiences shared by all participants. We are also grateful to Alexa Weinstein for editorial contributions and support in this work. Funding for this project was provided by Multnomah County Health Department. We wish to express our gratitude to the Future Generations Collaborative and CityMatCH and the Prevention of Substance-Exposed Pregnancies Collaborative, who provided expert technical assistance and guidance in the beginning phases of this work. 


\begin{abstract}
We examined the multidimensional cycle of fetal alcohol spectrum disorders (FASD) in the socio-cultural, historical, and interpersonal context of trauma shared by American Indian and Alaska Native (AI/AN) peoples living in Multnomah County, Oregon. This case study was guided by Evans-Campbell's (2008) multilevel framework of historical trauma and used community-based participatory research methods. Our study explored knowledge and attitudes about FASD, perspectives on FASD risk factors, and culturally relevant approaches to FASD prevention. Effective, multiple-level FASD prevention approaches for AI/ANs may include prioritizing Indigenous culture, supporting intergenerational cohesion, focusing on non-stigmatic healing of traumas, and authentically engaging community knowledge.
\end{abstract}

Keywords: American Indian/Alaska Native (AI/AN), fetal alcohol spectrum disorders (FASD), patient engagement, psychological trauma, historical trauma 


\section{An Indigenous Framework of the Cycle of Fetal Alcohol Spectrum Disorder Risk and Prevention Across the Generations: Historical Trauma, Harm and Healing}

Among American Indians and Alaska Natives (AI/ANs), reducing the occurrence of fetal alcohol spectrum disorder (FASD) is a major public health priority (Office of Disease Prevention and Health Promotion, 2015). FASD is a term used to describe a range of lifelong physical, cognitive, and behavioral impacts that can occur in an individual prenatally exposed to alcohol (Chudley et al., 2005; Sokol, Delaney-Black, \& Nordstrom, 2003). The lifelong health impacts of FASD range in severity and include learning and memory disorders, hyperactivity, distractibility, attention deficit, impulsivity, and difficulty processing information with sensorial impacts (Mattson, Crocker, \& Nguyen, 2011). Behavioral health issues co-occurring with FASD may include alcohol and drug addiction, mood swings, depression, and anxiety (Hellemans, Sliwowska, Verma, \& Weinberg, 2010). The current primary way to prevent FASD is to eliminate maternal alcohol consumption during pregnancy (Sarche, Fleming, \& Spicer, 2011). In general, the success of programs to reduce the incidence of alcohol-exposed pregnancies has been limited in AI/ANs (Montag, Clapp, Calac, Gorman, \& Chambers, 2012; Montag et al., 2015; Rentner, Dixon, \& Lengel, 2012). The inability to successfully prevent FASD among AI/ANs may be explained in two ways.

First, health programs and interventions targeting AI/ANs suffer from low engagement (Buchwald et al., 2006; Hodge, Weinmann, \& Roubideaux, 2000). Some AI/ANs disengage from such efforts due to medical mistrust (Goins, Garroutte, Fox, Dee Geiger, \& Manson, 2011; Guadagnolo et al., 2009), and to discrimination within a health care setting (Gonzales, Harding, Lambert, Fu, \& Henderson, 2013). Among AI/AN women, these beliefs emerge from and persist 
due to a deep legacy of medical maltreatment which includes sterilization without consent and child welfare removal (Carpio, 2004; Lawrence, 2000). This history further influenced by concerns about being negatively stigmatized and stereotyped (as a drunken Indian, for example), which lead to AI/AN women's concern that they will lose their child if they disclose that they are consuming or have consumed alcohol during their pregnancy (Poole \& Isaac, 2001).

Second, health programs in general have been designed to promote behavioral change by targeting individuals and often fail to consider the broader context that influence factors important to modifying and sustaining lifestyle changes (Airhihenbuwa, Ford, \& Iwelunmor, 2013; Jernigan, Jacob, \& Styne, 2015). Most FASD prevention and intervention programs have similar limitations and fail to consider FASD risk behaviors from a multidimensional perspective that takes sociocultural factors and systemic inequalities into account (Cunningham, 1993; May \& Gossage, 2011; Salmon \& Clarren, 2011; Warren, Hewitt, \& Thomas, 2011). Engagement problems cannot be explained solely on the level of individual behavior; the medical mistrust of $\mathrm{AI} / \mathrm{AN}$ patients exists in a complex multidimensional context that is rooted in historical, intergenerational, and contemporary traumas experienced by AI/ANs, and that also contributes to behavioral and perinatal health inequities.

Researchers are beginning to consider the links between trauma, health behaviors, and subsequent health outcomes for AI/ANs (May \& Gossage, 2011; Walters \& Simoni, 1999; Walters, Simoni, \& Evans-Campbell, 2002). For example, some scholars describe alcohol use among AI/ANs as a result of complex sociocultural factors and systemic inequalities, which have caused harm and trauma that continues to impact AI/ANs today (Evans-Campbell, 2008; Shahram, 2016; Szlemko, Wood, \& Thurman, 2006). Building on this understanding, more research is framing AI/AN health in intergenerational terms and suggesting a shift away from the 
dominant medical model, which may use a shame-and-blame approach that treats FASD risk solely as an individual-level problem (Hanson \& Pourier, 2016; Ospina, Moga, Dennett, \& Harstall, 2011). If FASD risk behaviors are generated on multiple levels, then programs which target only individual-level factors will always be limited in impact and effectiveness. A sole focus on the individual level also reflects a general failure of the health care system to consult with, and consider the needs of, the underrepresented communities it serves.

Most health care programming available to AI/ANs has been designed without factoring in community perspectives and experiences (Harden \& Oliver, 2001; Blue Bird Jernigan et al., 2015; Mehl-Madrona, 2000). These deficiencies also characterize many FASD intervention programs (George et al., 2007; Masotti et al., 2006; Mehl-Madrona, 2000; Mercier, 2014; Poole, 20109). To overcome the limitations of existing programs - low engagement and failure to consider and value sociocultural factors and systemic inequalities — and to achieve therapeutic goals for FASD prevention that emerge from culturally centered and non-stigmatizing approaches, researchers have emphasized the need for culturally responsive health programs. They recommend that these programs be designed to simultaneously target multi-level factors, addressing the problem of FASD without exclusively targeting individual-level behavior (Parker, Maviglia, Lewis, Phillip Gossage, \& May, 2010). To inform such programs, more research is needed to understand the broader context of the sociocultural factors that influence FASD risk, and the role of systemic inequalities (Mehl-Madrona, 2000; Parker et al., 2010). This information is especially needed among urban AI/ANs who access health and social services outside the largely reservation-based Indian Health Service (Rutman, Phillips, \& Sparck, 2016), and such programs may be lacking in important ways that result in failure to consider and prioritize the needs of AI/ANs. 
Therefore, this article describes a case study conducted in Portland, Oregon that sought input from one urban AI/AN community about the best ways to advance health equity and positive birth outcomes by reducing substance-exposed pregnancies for this population. The purpose of this case study is to explore knowledge and attitudes about FASD, perspectives on FASD risk factors, and culturally relevant approaches to FASD prevention.

\section{Theorical Framework}

Evans-Campbell's (2008) multilevel framework of historical trauma guided our study design and analysis. Evans-Campbell's framework articulates the need for researchers to examine AI/AN health in terms of communal and historical context. For example, she argues colonial trauma, is an important concept, referring to "colonial practices to colonize, subjugate, and perpetuate ethnocide and genocide against contemporary American Indian and Alaska Native peoples and nations" (p. 335). Thus, poor AI/AN health outcomes are not simply an individual problem, but rather always connected to the historical and communal contexts in which AI/ANs live. While Evans-Campbell draws from a broader literature on historical trauma, her multilevel framework is the first to articulate that stressors and health problems of AI/ANs rooted in colonial trauma needs to be examined in terms of individual, family, and community connections. We apply Evans-Campbell's multilevel framework to our study of FASD prevention among AI/ANs. In doing so, we avoid individualized public health approaches that victim-blame or view the issue in terms of an individualized behavioral health problem. Instead, we utilize a multilevel framework that can enrich our understanding of FASD in terms of historical and contemporary individual, family, and community dynamics among AI/ANs.

\section{Methods}


Our project utilized community-based participatory research (CBPR) to understand FASD among AI/ANs. CBPR is a widely-used methodological approach in health studies that seek to honor community voice and perspective, privilege underrepresented community perspectives, and power-share between researchers and research participants. Doing CBPR is a way of bridging "science and practice through community engagement and social action" (Wallerstein and Duran, 2010, p. S40). Because our study engages an underrepresented perspective (AI/ANs) and a potentially stigmatizing topic (FASD), we sought to build a respectful process that honored the wisdom of the community, the expertise of culture bearers, and to collaboratively move forward in a research project that would help eradicate the hierarchies between university and community that too often disempower community voice and perspective. We did so by designing the project with community feedback, employing community-nominated co-researchers who were valued as experts on community processes and cultures, and by sharing results, asking for feedback, and being available, on a weekly basis, for any questions or comments related to the project. All of these components of our CBPR process are effective equity-focused approaches in addressing health research challenges among marginalized populations (Wallerstein and Duran, 2010).

During the summer of 2014, the Future Generations Collaborative (FGC) conducted eight focus groups (referred to hereinafter as "forums") each lasting 1-1.5 hours. The FGC is a unique collective of organizations that serve AI/ANs, including AI/AN community members, community health and social services providers, County and State Health departments, and Portland State University. Collectively, the FGC is committed to reducing substance-affected pregnancies within the local AI/AN community in Portland, Oregon. 
Seventy-four members of the Portland Metro AI/AN community who self-identified as AI/AN participated in the forums. The forums were comprised as follows: two groups of young men $(n=37)$ and four groups of young women aged 15-24 (n=61) (hereinafter "young adults"), one mixed-gender group aged 25-54 (n=19) ("adults"), and one mixed-gender group of elders aged 55 and older (n=24) (“elder adults"). Eligibility criteria for this study were: 1) at least 15 years of age, 2) able to speak and read English, 3) self-identifying as AI/AN, 4) currently residing in Multnomah County, and 5) able to provide informed consent. Recruitment was led by research assistants who are trusted members of the AI/AN community. Information about the study was posted on the FGC Facebook page and key locations within community-based agencies that serve AI/ANs. The forums were held in locations known and trusted by AI/AN community members. Food, public transportation assistance, and childcare were provided at the time of the forums, but other incentives were not offered.

In keeping with community-based participatory research methods (Burhansstipanov, Christopher, \& Schumacher, 2005), the forums were facilitated by a team of community-member research assistants who were trained in community organizing, qualitative interviewing techniques, and human subjects research. The research assistants were individuals who selfidentified as Native American. Each had personal or family ties to the Native American community. None had previous research experience. In addition to training on research methods and completing the National Institutes of Health human subjects research training, they received capacity building to gain awareness about the pathways through which historical and intergenerational trauma correlated to poor mental health outcomes and harmful stress responses such as addictions, and had opportunities to develop personal, cultural and collective ways to engage in personal and community healing. As the group developed trust, relationships and 
shared capacity, they became more deeply engaged in the development of the research protocols, including co-developing the interview guide and facilitation process, co-authoring the IRB proposal, and leading recruitment of study participants. The research assistants facilitated the forums, supported data analysis, and provided a formal presentation of the findings of this study back to the community (Christopher, 2005).

Likewise, the principal researchers' included trusted Indigenous women with extensive experience working in partnership with Indigenous communities. All of their work uses CBPR principles and emphasizes understanding health inequities among Native Americans, especially related to historical and intergenerational trauma, racism, and addressing them through interventions that center socio-cultural solutions and draw on community strengths and wisdom to overcome the harmful impacts of unhealed trauma; one is the Executive Director of a acclaimed non-profit organization that serves Indigenous peoples and agencies that serve them, guided by the vision that "through the gifts of our ancestors...prayer, song, ceremony, language, and courage... Native people will live in balance and wellness, ensuring a rich cultural legacy for our future generations", and one is a $\mathrm{PhD}$-level Indigenous researcher working in a tenure-track appointment, in the School of Public Health, within a university setting. Other team members included a PhD-level Indigenous Maternal and Child Health epidemiologist and non-Native Health Educator from the local country health department.

Forums were conducted using a structured format that included an agenda, narrative, and interview guide that was guided by Evans-Campbell's multilevel framework of historical trauma (Evans-Campbell, 2008). The interview guide was designed to explore topics to help inform the following three issues: what is the relationship between substance use, reproductive health practices, and historical and intergenerational trauma in the urban AI/AN community?; What 
factors promote resilience, strength, healing, and are protective against substance-exposed pregnancies among members of the urban AI/AN community?; and, How can these communitydefined risk and protective factors best inform action steps that promote healthy pregnancy and also reduce substance exposed pregnancies in the community? Example questions used in the interview guide include: a.) What are your family and community stories and traditions around healthy pregnancy?, b.) what do you think protects against substance use before, during, and after pregnancy, c.) what do you think leads to substance use before, during, and after pregnancy?, d.) what supports and promotes healthy outcomes for our future generations?, d.) what prevents healthy outcomes for future generations?

Informed consent was obtained prior to participation, and an assent form was also collected from the parent/guardian of any participant under 18 years of age. In response to community preference, the forums were not audio recorded. Instead, extensive notes were taken by hand by two different assistants from the research team, to capture the information shared during the sessions. Data collection at the forums was facilitated by a moderator and two assistant notetakers who were recruited from the cohort of community research assistants trained for this particular project.

Directly following each forum, note takers and facilitators debriefed what was heard, what was felt, what was seen. This debrief was incorporated into the evaluation notes and combined into one master electronic document to be coded for themes and analyzed using a grounded theory approach (Strauss \& Corbin, 1990). The note takers reviewed the data, and identified major themes, and developed a thematic a coding system for analysis. Next, the analysis and coding system was shared and reviewed by the PIs of this project for further analysis, and verification. Then those involved with analysis gathered together to discuss, review, 
contextualize and integrate the themes. Themes were agreed upon via consensus among those conducting the analysis, using an iterative process to determine the final themes, as well as emergent themes and quotes to illuminate the concepts and meanings of the themes. Finally, in keeping with principles of community-based participatory research, the results of the forums were presented back to the entire research team, including the trained community-research assistants as the first level of community validation. A report was then presented to participants that were involved in the actual forum session, to complete the second level of community validation. These presentations were facilitated by the community-member research assistants to validate the key findings and gain permission to share the results of the forums with the broader community. Finally, a report was then presented to complete the third level of community validation among the broader community that may not have been involved with this particular project.

Approval to conduct this study, as well as study protocol, was obtained from the appropriate institutional review boards, including those of the Northwest Portland Area Indian Health Board, Portland State University, and the Multnomah County Health Department. While the research team did not offer direct 1-1 follow-up to ensure that emotional supports were offered to individuals following their participation, the community forums were facilitated using a traumainformed approach. For example, each forum was opened and closed in a culturally respectful way to help build trust, build community and to send participants home in a good way. In addition, the facilitator guide asked questions about intergenerational trauma first and ended with personal and community healing strategies, to end on a discussion that was strength-based. 


\section{Results}

Three interconnected themes were identified from the data: (1) knowledge of and experiences with FASD, (2) the cycle of FASD risk, and (3) culturally specific solutions to prevent FASD and support those affected by FASD. The findings for these categories are presented in the section below.

\section{Theme 1: Knowledge of and Experiences with FASD}

Participants across the eight groups were aware that FASD was caused by exposure to alcohol during prenatal development. Several of the young-adult men and women also believed that FASD was caused by the father's substance abuse at the time of conception, reporting that alcohol "affected the sperm," and there is some evidence to support this claim (Lee et al., 2013). Participants stated that their knowledge about FASD was gained through various mechanisms including: personal experience, acquired knowledge through school, and stories passed along within families and shared between friends. Thus, participants insisted on the social and relational aspects of FASD as important, in contrast to a purely behavioral understanding of FASD, which most often attempts to isolate a pregnant woman's drinking behavior as the sole "cause" of FASD.

Most of the participants stated that they knew someone with FASD. Others had witnessed pregnant women in their community drinking alcohol and/or member(s) of their own familyincluding in some cases their own mothers-drinking while pregnant. Additionally, some of the women courageously discussed their own alcohol use during their pregnancies. An adult woman shared: "I've had experiences drinking with two women. Both were pregnant and both drank. I was drinking, too.” 
Men from two age groups (young adults and adults) indicated that they themselves were exposed to alcohol prenatally and are now affected by FASD. Some reported varying degrees of autism and Attention Deficit and Hyperactivity Disorder, which they attribute to FASD-related health conditions as a result of their mothers "drinking and drugging" while pregnant with them.

Participants across all eight groups suggested that they knew people in their family or within the Native community who they suspected to have FASD. Generally participants made this determination because they witnessed certain behaviors or developmental challenges that they associated with FASD, including "showing hyperactivity, lacking common sense, thinking abnormally, misunderstanding cause and effect, and having ADHD." Highlighting these points, one of the male young adults stated: "Some of my friends have FASD. They look normal but on the inside they are not all there. You don't have to look abnormal. It's mental, and they will have to live with that their whole life."

With regard to identifying those with FASD, both male and female adults expressed concerns about stereotyping of AI/ANs, and over-association of FASD with Native communities, within service agencies and society at large. In their opinion, staff sometimes wrongly assume that children have FASD because their parents are AI/AN. One participant in this age group stated, “In foster care it's like, 'Oh, he must act that way, he's an Indian, he must have FASD.' " Such data indicate a perception among AI/ANs that state agencies, such as the foster care system, stereotype $\mathrm{AI} / \mathrm{ANs}$ in relation to FASD.

Finally, participants from all groups agreed that FASD is a serious issue for the community. A young-adult woman described FASD as "a concern because we want healthy babies and healthy kids in the community." Many participants associated the problem of FASD with the 
well-being of their own families, their community, and "future generations." Another youngadult woman stated:

My mom drank with me and my sister. If I do end up having children, I am definitely not going to do that. I will make it so that people won't want to do that, I want to break the cycle.

Overall, the participants in this study demonstrated extensive and detailed knowledge about FASD, and considerable personal experience with its damaging effects.

\section{Theme 2: The Cycle of FASD Risk}

Participants from all eight groups discussed a wide range of factors that they believe contribute to substance-exposed pregnancies within the Native community. The primary factors discussed were: social norms related to drinking; coping in response to stress and hurt from historical, intergenerational, and contemporary traumas; and issues of medical mistrust that make it difficult for women to freely disclose and discuss alcohol use during pregnancy. Taken together, these comments described a complex cycle through which FASD is cultivated and perpetuated. This cycle supports Evans-Campbell's (2008) previous work to theorize the impacts and effects of historical trauma among AI/AN peoples across multiple levels: individual, family, and community.

Across all groups, participants related personal experiences that led them to drink alcohol, and discussed why this behavior was prevalent in their own lives. Among young adults of both genders, some shared that they (or their friends) drank to have fun and to connect socially and fit in with their friends. For example, young-adult women indicated that drinking made them feel older and more rebellious. Many of the young-adult participants felt pressure to drink, especially when around others who were also drinking alcohol. An adult participant voiced the belief that 
"young people drink because of peer pressure, and as a way to respond against bullying that they may encounter."

Across all eight groups, most participants stated that alcohol use is common in their families and social networks. They described drinking as a social norm that is perpetuated by modeling the same behavior. According to an elder adult, "Drinking and alcoholism was the norm growing up, that's what you saw and that's what you did." Participants further characterized drinking as a "cycle shared across generations." Another elder adult recounted:

I grew up in an alcoholic home, my dad was alcoholic. It was part of manhood to drink to become a man. I started when I was 13. Alcohol has devastated our people. I grew up thinking it was OK.

\section{Coping}

When participants began to explain the underlying reasons for the pervasiveness of alcohol use in their communities, they turned to the subject of coping. According to participants, drinking helped them to escape stress and/or emotional pain. Both young-adult men and women reported drinking alcohol to "drown out the pain," and to help them "forget things, numb pain and depression, and to make memories go away." Other adults suggested that they use alcohol as a tool to "get away from things in their lives."

While participants did not see drinking alcohol as a long-term solution to emotional pain, they nevertheless expressed empathy for family and community members who turned to alcohol to numb the pain in their lives. One participant expressed it this way: "People drink because they have pain inside and even if it helps for a couple of hours, that couple of hours means a lot." At the same time, participants across all eight groups shared that even though drinking was prevalent in their lives, seeing people from their families and community who were drunk caused 
“depression, shame, and embarrassment." Participants described alcohol use as a coping mechanism used to ameliorate emotional pain, but they also described it as a cause of further emotional pain — a cause of depression, shame, and behavior that leads to more emotional pain.

Many participants described the emotional pain that leads to drinking as being caused by events in the past. Individuals from all eight groups spoke about "unhealed hurts, emotions, and worry." This historical pain referred to individual experience but also to the collective experiences of Native people. An elder adult believed that her parents drank "to forget their hard lives when they were growing up and because of death, boarding school, moving from reservation to urban areas, and dealing with step-parents and step-children.” One young-adult man stated, "I think alcohol is historical trauma. What do we feel when we get drunk? Anger. Because that's what happens, you get angry and you fight."

\section{Historical events/traumas}

Historical trauma was described on the communal level as well as the individual level. Many viewed alcohol use as a result of colonization and oppression, and some believed that alcohol was "intentionally introduced into Native communities by the colonizer to harm them" as a weapon of genocide and instability. An adult participant stated, "I think what's going on here is a 100-year-old war that is meant to destroy us. That's why the colonizers brought it [alcohol] here and it's hitting us from all directions."

Participants across the eight groups described alcohol use within Native communities as a consequence of historical events that were traumatic, while simultaneously viewing it as a source of contemporary trauma. In fact, they described alcohol use as one of the primary mechanisms through which historical trauma is passed down intergenerationally, to become contemporary trauma. A male adult participant stated: 
In my family we have multi-generational alcohol and drug addiction. On both sides we have alcohol. We have a lot of deaths from alcohol and the pain from it, my mom and dad, aunts, uncles, a lot of family in prison. I know it's the same for a lot of my friends. In the cycle described by these participants, historical trauma causes emotional pain, which causes alcohol use among the older generation, which causes emotional pain for the next generation. When the younger generation turns to alcohol as a socially normalized habit, a coping mechanism, and a treatment for emotional pain, the cycle is perpetuated. In this way, historical trauma becomes contemporary trauma through the mechanism of alcohol use.

Participants spoke about experiencing contemporary trauma in their everyday lives in multiple forms: alcohol-related domestic violence, removal of children from their homes into the foster care system, lateral oppression, violence toward others, loss of land and language, and isolation and disconnection from cultural values and community. They shared that these everyday experiences were exacerbated by common experiences of racism and stereotyping, as well as a failure of the dominant culture to stand up against these injustices. Together, participants described many ways in which the historical trauma that was passed down intergenerationally has manifested in their lives as contemporary trauma and harms them.

\section{Family Impact and Influence}

Finally, participants located FASD in the larger cycle they were describing, explaining how all of these factors increase the risk of substance-exposed pregnancies. An adult participant explained the emotional pain caused by FASD in this way: "My friend is affected by FASD. He's struggling spiritually and otherwise. He's always bringing himself down. He quit dancing. He quit singing. He quit sweat. He said, 'I can't do it anymore.' " 
The participants in this study described an interwoven cycle within families that helps to explain the origins of FASD risk, the lack of resources for families to address their needs regarding FASD, and the subsequent negative impact on relationships. Collectively, they described the following cycle. When a family can't raise its family member(s) affected by FASD in ways that are nurturing and healthy, this causes pain and guilt. The families carry additional guilt, shame, and denial about the drinking that occurred while the mother was pregnant, which then raises additional concern that the drinking could lead to the child being taken away and placed in the foster care system, which causes more pain and hurt. This is linked to the stories told within families and across generations about experiences of children being taken away, and ultimately this cycle can lead to more alcohol use.

\section{Mistrust with the Healthcare System}

The specific ways in which the cycle described by participants increases the risk of substance-exposed pregnancies were further illuminated when the women in the study discussed the complexities of disclosing alcohol use during pregnancy after it occurs. Barriers related to disclosure included personal experiences of being treated unfairly in health care, concern about being treated according to the strong stereotype "that all Natives drink and are alcoholics," and fear of losing their child due to being labeled "an unfit mother." These responses were shared by young-adult and adult female participants, and often the sharing was based on the respondents' own personal experiences.

A number of stories were told in which a woman used alcohol after becoming pregnant but before finding out about the baby. One woman told her own story this way: 
I was taught to stay away from bad influences. I didn't find out until I was 3 months pregnant. I didn't know what to do. I just stayed away from everyone. I moved here when I was 5 months pregnant and I prayed that he would come out healthy.

In many cases, women who could have sought medical help and advice after mistakenly drinking while pregnant did not do this, due to shame, medical mistrust, and isolation.

Many participants expressed general fear and uncertainty about engaging with health care, deriving from their knowledge about the historical experiences of Native people.

Additional factors that made it difficult for participants to trust and engage in health care resulted from inconsistent health messages that conflicted with their own knowledge and experience. For example, both young-adult women and men reported being told by health care providers that "drinking alcohol was helpful in nursing." A young-adult man stated that his girlfriend was told by her doctor to "drink a beer to help her milk come in," and a young-adult woman heard that "drinking wine during pregnancy is OK and will not harm the baby." These messages conflicted with the participants' extensive knowledge of and experience with FASD, but the conflict was not able to be resolved within the context of medical mistrust.

\section{Theme 3: Solutions to Prevent FASD and Support Those Affected by FASD}

Participants from across the eight groups articulated a vision of a healthy Native community in which there are opportunities for individual- and community-level healing. Many of the participants believed that strengthening existing connections and creating new connections, to each other and to cultural practices, offered the promise of healing. Opportunities for healing cited across all the groups included: drumming, sweat lodge, talking circles, beading, and sharing traditional cultural practice across generations with regard to pregnancy, birth, parenting, healthy relationships, and understanding the sources and role of trauma. These efforts were further 
described as being most impactful when they draw from cultural and community-based strengths of positivity and resilience, and least helpful when they stigmatize or shame. Articulating the belief shared by many participants that cultural activities are central to healing, one young-adult woman explained: "We want our culture to be brought out more. We have to help our young people get into their culture. If you can't communicate with them they are going to get away, just like some of us did."

Participants from across the eight groups discussed the importance of families creating a healthy and supportive home environment, because alcohol use and abuse harms the family as a whole as well as the individuals in it. Echoing a common sentiment, one participant asserted that "healing is needed and the whole family needs to be involved." An older adult also stated:

This change will in part come from adults taking a stand, and perhaps being of service to the community by mentoring youth, bringing them to meetings and community activities, to be someone that the youth can look up to as a role model, be a positive influence and do the right thing.

Finally, participants described the necessity of changing health and social services programs so they create spaces that are culturally safe and non-stigmatizing, and so they demonstrate a deep and authentic valuing of AI/ANs throughout all programming, policies, and services. This can be accomplished by embedding AI/AN cultural values within health and social service programs that serve AI/AN women and families. Health and social services systems also need to acknowledge the role of government-led health care programs in causing and perpetuating traumatic experiences for AI/ANs. Participants across all groups stated that they want more opportunities for culturally based health care services, and more supportive and non-judgmental 
programs that do not stereotype Native people. Most importantly, participants want to feel that they and their cultures are valued by health and social services systems.

\section{Discussion}

This study engaged in community-based participatory research to investigate the underlying risk factors of FASD from the perspective of a large and diverse AI/AN community. We found that participants had extensive knowledge of and experiences with FASD. We also found that participants articulated a complex multidimensional cycle through which FASD occurs and is perpetuated. These findings advance our understanding of FASD risk and consequences within an urban-based AI/AN population and support and extend what Evans-Campbell (2008) theorized in her work on historical trauma; AI/ANs view FASD as inherently connected to a multilevel framework of trauma and healing. Specifically, AI/ANs view culture as a protective factor that can help mitigate the harmful ongoing effects of colonization and colonial trauma within $\mathrm{AI} / \mathrm{AN}$ communities. Additionally, AI/ANs view "getting away from” Indigenous cultural traditions as damaging and increasing risk—at the individual, familial, and communal levels. We found that $\mathrm{AI} / \mathrm{AN}$ community members articulated an Indigenous framework for understanding FASD risk and perpetuation that fully integrates multilevel sociocultural factors and systemic inequalities. This framework suggests a model for effective prevention and programming that includes support for more effective integration of Indigenous cultural norms and better awareness and recognition of the role of historical and contemporary trauma in the behavioral and mental health of AI/AN peoples. These data can inform development of other prevention and social service approaches that seek to engage AI/ANs and improve health equity among AI/ANs.

Participants described a sophisticated, interconnected, and cyclical mechanism through which FASD risk occurs and is perpetuated and transmitted in the urban AI/AN community. This 
mechanism is described in Figure 1, "Indigenous Framework of the Cycle of FASD Risk and Prevention."

The framework shows that FASD occurs and is perpetuated in urban AI/AN communities as a result of the historical, contemporary, and intergenerational transmission of experiences considered to be traumatic. First, AI/AN people live with "blood memories" (Heart \& Horse, 2000) and everyday experiences of individual and community trauma (Evans-Campbell, 2008). In our study, historical traumas are described by participants as the long history of oppression by colonization, violence, and racism that still affects AI/AN people today, and this is similar to previous research (Ball \& O’Nell, 2015; Evans-Campbell, 2008; Evans-Campbell, Walters, Pearson, \& Campbell, 2012; Mercier, 2014; Soto, Baezconde-Garbanati, Schwartz, \& Unger, 2015; Walters et al., 2002). These traumas include historical instances of genocide, medical experimentation, and medical malpractice that were sanctioned by the state and reflected deeply entrenched institutional racism (U.S. Commission on Civil Rights, 2004).

Co-occurring and parallel processes occur when historical and contemporary traumas coincide, and families, communities, and cultural engagement are disrupted as a result. For example, AI/AN families who experience the contemporary trauma of displacement and forced disconnection when a family member is incarcerated may also re-experience the historical trauma of displacement and forced disconnection (i.e., removal of Native children from parents or displacement from land) (Evans-Campbell, 2008). Alcohol use is described as a coping mechanism which provides emotional relief from the cycle of trauma and the disruption of familial, community, and cultural ties. This coping mechanism, also described by participants as self-medicating, is often passed across the generations, further perpetuating intergenerational and historical trauma. Participants describe a cycle in which historical trauma is passed down from 
past generations to current generations through the mechanism of alcohol use, within families and communities, as cited in previous research (Walters \& Simoni, 1999).

Participants also describe other mechanisms through which sociocultural factors and systemic inequalities generate contemporary trauma, including the lack of consideration and prioritization of AI/AN health and wellness, and the lack of culturally specific approaches that integrate Indigenous ways of knowing and doing into service provision and program design. Underrepresentation of AI/AN people within service systems may increase the likelihood that AI/AN patients will experience discrimination within health care settings and subsequently disengage. Similar deficiencies and their contribution to AI/AN health disparities have been previously cited in the published literature (Blue Bird Jernigan et al., 2015; Gone, 2013a, 2013b; Gonzales et al., 2013; Parker et al., 2010). This study, however, situates these health care issues within a larger cycle that provides new understanding of the multidimensional context of FASD risk.

Our third finding, that members of this community provided specific recommendations for FASD prevention that would be effective for them, also merits further discussion. Participants in this study conceptualized FASD prevention not simply as the provision of services, but as a holistic process for healing in which culturally relevant services are but one component of the model. This is an important departure from dominant cultural models of FASD prevention, including many best-practice and evidence-based models in use nationwide. Community members want opportunities for healing at multiple levels, and they expect that systems will also do their own learning and healing. They point to inequities in systems serving AI/ANs and recognize that, as part of an interconnected community, systems too must address ways in which they are perpetuating trauma; the work is not to be done by individuals and families alone. 
Our data show that healing should be broad in scope, and should span across generations. For example, while learning about the risks of FASD and how alcohol can impact a developing fetus, participants must also learn about the use of alcohol in both its historical and contemporary contexts, as a weapon of colonization, and consider how it still is harming AI/ANs. They must understand what historical and intergenerational trauma are and how they affect health over time, and have the opportunity to talk honestly about the fear, grief, shame, and pain that alcohol and the origins of alcohol use have caused AI/AN people and families, especially among people who drank while they were pregnant. Similar inquiry is also needed within systems that serve AI/AN peoples. Such inquiry should be expected not only at the system level, but also among those working within such systems. Examples of subjects of inquiry include understanding the nature and scope of implicit bias among the professionals within the healthcare system, how to be a helpful [decolonized] alley, and the unintended harm that may result from the lack of such inquiry. Participants suggested that education and capacity-building should happen within cultural contexts, such as through the sharing of cultural practices and traditions, like beading or those related to pregnancy and health. They suggested that providers should also be educated, so that they share in this deeper understanding about the role of alcohol in perpetuating the intergenerational trauma of colonization, carrying the damage of the past forward and continuing to harm AI/AN people in the present. These findings correspond to those in the published research that explores similar issues (Shahram, S. Z., Bottorff, J. L., Kurtz, D. L. M., Oelke, N. D... (2016)

In sum, participants did not see substance use as an isolated incident, but as a behavioral outcome resulting from the interconnected cycle of historical trauma, reduced social and familial support, increased substance use, and contemporary trauma. Therefore, an FASD prevention 
program in an urban AI/AN community is unlikely to be effective if it focuses solely on: (1) intervening at the time of pregnancy; (2) preventing a substance-exposed pregnancy through the practice known as screening brief intervention and referral to treatment (SBIRT); and (3) prescription of a long-acting contraceptive method if substance use cannot be prevented while a woman is sexually active. Approaches like the one just described fail to address the root causes of FASD risk - historical, contemporary, and intergenerational traumas. They also fail to support people already living with an FASD — a critical oversight, since youth and adults with FASD are at highest risk for having their own substance-exposed pregnancies (Floyd, Ebrahim, \& Boyle, 1999). Finally, FASD prevention programs that do not address the role of family, community, and culture in creating resiliency, positive cultural norms, and strong family and community ties will likely not achieve or sustain reduced rates of FASD.

Using the recommendations of this community, our study provides recommendations for designing programs that avoid the previous limitations of low engagement, individual-level focus, and lack of community input. The experiences summarized in this paper further substantiate the need for $\mathrm{AI} / \mathrm{AN}$ involvement in the development of health programming, including implementation and evaluation of prevention programs that seek to reduce incidence and prevalence of FASD. Participants challenge practitioners in public health, health care, and social services to carefully consider and attend to the effects of historical and contemporary trauma, including structural racism, on patient engagement and behavioral health outcomes. Namely, they challenge us to expand our thinking of what "trauma-informed approaches" mean. An expanded understanding of this emerging practice would encompass more than clientprovider interactions; it would also consider the ways that our systems create contemporary 
trauma though our own policies and practices, or ignoring its profound effect on engagement and behavioral health outcomes.

It is also clear from these data that the role of family, community, and culture is essential to addressing FASD. Socio-cultural (re)connection and trauma healing is a critical pathway to better health. Importantly, participants articulated a holistic model of FASD prevention. In this model, FASD prevention can function as a mechanism to promote a healthy and thriving Native community that is connected to culture, that promotes strong intergenerational bonds, and that views pregnancy not as an isolated experience for a woman but as a sacred event to be welcomed and invited into a family and community. Participants view Indigenous culture as a protective factor, and articulate that it is key to community wellness; these are views that are articulated in the broader literature about Indigenous community well-being (Jacob et al., 2013).

\section{Limitations}

Our findings should be considered in light of potential limitations. For example, our study utilized a convenience sample. Such sampling contributes to various biases, including selfselection bias, which may have occurred if the sample over-represents individuals who are motivated to participate. Participation may also have been influenced by personal relationships between the participant(s) and member(s) of the research team. In spite of the potential for such bias, this study featured a large and diverse sample, comprised of men and women of varying ages, and representing a wide range of experiences and perspectives. Within a research framework that centers Indigenous methodologies, however, personal relationships are valued and identified as a critical component for trust building and overcoming mistrust of research. For this particular study, these relationships may contribute in profound and positive ways that 
resulted in successful recruitment and rendering data that was rich and meaningful to meet the needs of those most impacted and yet, widely under- represented in research.

\section{Conclusion}

We suggest that efforts to address FASD among urban AI/ANs incorporate an understanding of trauma, and utilize culturally-informed methods. According to our study’s participants, such efforts should: align with and emerge from community values, promote healing, consider the broader context that influences behaviors, and reflect the community's understanding that FASD risk behaviors are inextricably linked with historical and contemporary trauma. Our participants framed FASD as a social-cultural-historical phenomenon that impacts the entire community. This understanding of FASD transcends a limited public health or medical perspective, which might view FASD as solely a matter of individual-level behavior. In conclusion, this work draws on community and cultural strengths in an effort to reduce the occurrence of substance-exposed pregnancies, and encourages transformational changes in systems that serve AI/AN peoples to promote a healthy and thriving community and future generations. 


\section{References}

Airhihenbuwa, C. O., Ford, C. L., \& Iwelunmor, J. I. (2013). Why culture matters in health interventions lessons from HIV/AIDS stigma and NCDs. Health Education \& Behavior, 1090198113487199.

Ball, T., \& O’Nell, T. D. (2015). Square pegs and round holes: Understanding historical trauma in two Native American communities. Culture and PTSD: Trauma in Global and Historical Perspective, 334.

Blue Bird Jernigan, V., Peercy, M., Branam, D., Saunkeah, B., Wharton, D., Winkleby, M., .. . Buchwald, D. (2015). Beyond health equity: Achieving wellness within American Indian and Alaska Native communities. American Journal of Public Health, 105(S3), S376S379. doi: 10.2105/ajph.2014.302447

Buchwald, D., Mendoza-Jenkins, V., Croy, C., McGough, H., Bezdek, M., \& Spicer, P. (2006). Attitudes of urban American Indians and Alaska Natives regarding participation in research. Journal of General Internal Medicine, 21(6), 648-651.

Burhansstipanov, L., Christopher, S., \& Schumacher, S. A. (2005). Lessons learned from community-based participatory research in Indian country. Cancer Control, 12 Suppl 2, 70-76.

Carpio, M. V. (2004). The lost generation: American Indian women and sterilization abuse. Social Justice, $31(4$ (98), 40-53.

Christopher, S. (2005). Recommendations for conducting successful research with Native Americans. Journal of Cancer Education, 20(1 Suppl), 47-51. doi: 10.1207/s15430154jce2001s_11 [doi] 
Chudley, A. E., Conry, J., Cook, J. L., Loock, C., Rosales, T., \& LeBlanc, N. (2005). Fetal alcohol spectrum disorder: Canadian guidelines for diagnosis. Canadian Medical Association Journal, $172(5$ suppl), S1-S21.

Cunningham, P. J. (1993). Access to care in the Indian Health Service. Health Affairs (Project Hope), 12(3), 224-233.

Evans-Campbell, T. (2008). Historical trauma in American Indian/Native Alaska communities: A multilevel framework for exploring impacts on individuals, families, and communities. Journal of Interpersonal Violence, 23(3), 316-338. doi: 10.1177/0886260507312290

Evans-Campbell, T., Walters, K. L., Pearson, C. R., \& Campbell, C. D. (2012). Indian boarding school experience, substance use, and mental health among urban two-spirit American Indian/Alaska Natives. The American Journal of Drug and Alcohol Abuse, 38(5), 421427. doi: $10.3109 / 00952990.2012 .701358$

Floyd, R. L., Ebrahim, S. H., \& Boyle, C. A. (1999). Observations from the CDC: Preventing alcohol-exposed pregnancies among women of childbearing age: The necessity of a preconceptional approach. Journal of Women's Health \& Gender-Based Medicine, 8(6), 733-736.

George, M., Masotti, P., MacLeod, S., Van Bibber, M., Loock, C., Fleming, M., . . Prince, E. (2007). Bridging the research gap: Aboriginal and academic collaboration in FASD prevention. The healthy communities, mothers and children project. Alaska Medicine, 49(2 Suppl), 139-141.

Goins, R. T., Garroutte, E. M., Fox, S. L., Dee Geiger, S., \& Manson, S. M. (2011). Theory and practice in participatory research: Lessons from the Native Elder Care Study. The Gerontologist, 51(3), 285-294. doi: 10.1093/geront/gnq130 
Gone, J. P. (2013a). A community-based treatment for Native American historical trauma: Prospects for evidence-based practice. Spirituality in Clinical Practice, 1(S), 78-94. doi: 10.1037/2326-4500.1.s. 78

Gone, J. P. (2013b). Redressing First Nations historical trauma: Theorizing mechanisms for indigenous culture as mental health treatment. Transcultural Psychiatry, 50(5), 683-706. doi: $10.1177 / 1363461513487669$

Gonzales, K. L., Harding, A. K., Lambert, W. E., Fu, R., \& Henderson, W. G. (2013). Perceived experiences of discrimination in health care: A barrier for cancer screening among American Indian women with type 2 diabetes. Women's Health Issues, 23(1), e61-e67.

Grossman, D. C., Baldwin, L.-M., Casey, S., Nixon, B., Hollow, W., \& Hart, L. G. (2002). Disparities in infant health among American Indians and Alaska Natives in US metropolitan Aareas. Pediatrics, 109(4), 627-633. doi: 10.1542/peds.109.4.627

Guadagnolo, B. A., Cina, K., Helbig, P., Molloy, K., Reiner, M., Cook, E. F., \& Petereit, D. G. (2009). Medical mistrust and less satisfaction with health care among Native Americans presenting for cancer treatment. Journal of Health Care for the Poor and Underserved, $20(1), 210-226$.

Hanson, J., \& Pourier, S. (2016). The Oglala Sioux Tribe CHOICES Program: Modifying an existing alcohol-exposed pregnancy intervention for use in an American Indian community. International Journal of Environmental Research and Public Health, 13(1), 1.

Harden, A., \& Oliver, S. (2001). Who’s listening? Systematically reviewing for ethics and empowerment. In S. Oliver \& G. Peersman (Eds.), Using Research for Effective Health Promotion. Buckingham: Open University Press. 
Heart, B., \& Horse, M. Y. (2000). Wakiksuyapi: Carrying the historical trauma of the Lakota. Tulane Studies in Social Welfare, 21(22), 245-266.

Hellemans, K. G. C., Sliwowska, J. H., Verma, P., \& Weinberg, J. (2010). Prenatal alcohol exposure: Fetal programming and later life vulnerability to stress, depression and anxiety disorders. Neuroscience \& Biobehavioral Reviews, 34(6), 791-807. http://dx.doi.org/10.1016/j.neubiorev.2009.06.004

Hodge, F. S., Weinmann, S., \& Roubideaux, Y. (2000). Recruitment of American Indians and Alaska Natives into clinical trials. Annals of Epidemiology, 10(8, Supplement 1), S41S48.

Jacob, M. M. (2013). Yakama rising: Indigenous cultural revitalization, activism, and healing. Tucson, AZ: University of Arizona Press.

Jacob, M. M., Gonzales, K. L., Calhoun, D., Beals, J., Muller, C. J., Goldberg, J., . . Howard, B. V. (2013). Psychological trauma symptoms and Type 2 diabetes prevalence, glucose control, and treatment modality among American Indians in the Strong Heart Family Study. Journal of Diabetes and Its Complications, 27(6), 553-557. doi: 10.1016/j.jdiacomp.2013.07.008

Jernigan, B. V., Jacob, T., \& Styne, D. (2015). The adaptation and implementation of a community-based participatory research curriculum to build tribal research capacity. American Journal of Public Health, 105(S3), S424-S432. doi: 10.2105/ajph.2015.302674 Lawrence, J. (2000). The Indian Health Service and the sterilization of Native American women. The American Indian Quarterly, 24(3), 400-419. 
Lee, H. J., Ryu, J.-S., Choi, N. Y., Park, Y. S., Kim, Y. I., Han, D. W., . . Ko, K. (2013). Transgenerational effects of paternal alcohol exposure in mouse offspring. Animal Cells and Systems, 17(6), 429-434. doi: 10.1080/19768354.2013.865675

Masotti, P., George, M. A., Szala-Meneok, K., Morton, A. M., Loock, C., Van Bibber, M., ... MacLeod, S. (2006). Preventing Fetal Alcohol Spectrum Disorder in Aboriginal communities: A methods development project. PLoS Medicine, 3(1), e8. doi: 10.1371/journal.pmed.0030008

Mattson, S. N., Crocker, N., \& Nguyen, T. T. (2011). Fetal Alcohol Spectrum Disorders: Neuropsychological and behavioral features. Neuropsychology Review, 21(2), 81-101. doi: 10.1007/s11065-011-9167-9

May, P. A., \& Gossage, J. P. (2011). Maternal risk factors for Fetal Alcohol Spectrum Disorders: Not as simple as it might seem. Alcohol Research \& Health, 34(1), 15-26.

Mehl-Madrona, L. E. (2000). Psychosocial prenatal intervention to reduce alcohol, smoking and stress and improve birth outcome among minority women. Journal of Prenatal \& Perinatal Psychology \& Health, 14(3/4), 257.

Mercier, A. (2014). Trauma-informed research and planning: Understanding government and urban Native community partnerships to addressing substance-exposed pregnancies in Portland, OR (Master's thesis). Portland State University, Portland, Oregon. http://pdxscholar.library.pdx.edu/open_access_etds/1803/

Montag, A., Clapp, J. D., Calac, D., Gorman, J., \& Chambers, C. (2012). A review of evidencebased approaches for reduction of alcohol consumption in Native women who are pregnant or of reproductive age. The American Journal of Drug and Alcohol Abuse, 38(5), 436-443. doi: 10.3109/00952990.2012.694521 
Montag, A. C., Brodine, S. K., Alcaraz, J. E., Clapp, J. D., Allison, M. A., Calac, D. J., . . . Chambers, C. D. (2015). Preventing alcohol-exposed pregnancy among an American Indian/Alaska Native population: Effect of a screening, brief intervention, and referral to treatment intervention. Alcoholism: Clinical and Experimental Research, 39(1), 126-135. doi: 10.1111/acer.12607

Office of Disease Prevention and Health Promotion. (2015). Maternal, infant, and child health objectives. In Healthy People 2020, 21.1-21.5.

Ospina, M., Moga, C., Dennett, L., \& Harstall, C. (2011). A systematic review of the effectiveness of prevention approaches for Fetal Alcohol Spectrum Disorder. In S. Clarren, A. Salmon and E. Jonsson (Eds.), Prevention of Fetal Alcohol Spectrum Disorder FASD: Who is responsible? (1st Ed., 99-335). Weinheim, Germany: WileyVCH Verlag GmbH \& Co. KGaA. doi: 10.1002/9783527635481.ch3

Parker, T., Maviglia, M. A., Lewis, P. T., Phillip Gossage, J., \& May, P. A. (2010). Psychological distress among Plains Indian mothers with children referred to screening for Fetal Alcohol Spectrum Disorders. Substance Abuse Treatment, Prevention, and Policy, 5, 22-22. doi: 10.1186/1747-597x-5-22

Poole, N. (2010). Bringing a women's health perspective to FASD prevention. In E. P. Riley, S. Clarren, J. Weinberg and E. Jonsson (Eds.), Fetal Alcohol Spectrum Disorder: Management and policy perspectives of FASD (First Ed., 161-174). Weinheim, Germany: Wiley-VCH Verlag GmbH \& Co. KGaA.

Poole, N., \& Isaac, B. (2001). Apprehensions: Barriers to treatment for substance-using mothers. British Columbia Centre of Excellence for Women's Health Vancouver. 
Rentner, T. L., Dixon, L. D., \& Lengel, L. (2012). Critiquing fetal alcohol syndrome health communication campaigns targeted to American Indians. Journal of Health Communication, 17(1), 6-21. doi: 10.1080/10810730.2011.585692

Rutman, S., Phillips, L., \& Sparck, A. (2016). Health care access and use by urban American Indians and Alaska Natives: Findings from the National Health Interview Survey (200609). Journal of Health Care for the Poor and Underserved, 27(3), 1521-1536.

Salmon, A., \& Clarren, S. K. (2011). Developing effective, culturally appropriate avenues to FASD diagnosis and prevention in Northern Canada. International Journal of Circumpolar Health, 70(4), 428.

Sarche, M. C., Fleming, C., \& Spicer, P. (2011). The prevention of fetal alcohol spectrum disorders in tribal communities. In M. C. Sarche, P. Spicer, P. Farrell, \& H. E. Fitzgerald (Eds.), American Indian children and mental health: Development, context, prevention, and treatment. Santa Barbara, CA: ABC-CLIO, 233.

Shahram, S. (2016). The social determinants of substance use for Aboriginal women: A systematic review. Women \& Health, 56(2), 157-176. doi:

$10.1080 / 03630242.2015 .1086466$

Shahram, S. Z., Bottorff, J. L., Kurtz, D. L. M., Oelke, N. D., Thomas, V., Spittal, P. M., \& Partnership, f. t. C. P. (2016). Understanding the life histories of pregnant-involved young Aboriginal women with substance use experiences in three Canadian cities. Qualitative Health Research. doi: 10.1177/1049732316657812

Smith, L. T. (2013). Decolonizing methodologies: Research and indigenous peoples. Zed Books Ltd. 
Sokol, R. J., Delaney-Black, V., \& Nordstrom, B. (2003). Fetal alcohol spectrum disorder. Journal of the American Medical Association, 290(22), 2996-2999. doi: 10.1001/jama.290.22.2996

Soto, C., Baezconde-Garbanati, L., Schwartz, S. J., \& Unger, J. B. (2015). Stressful life events, ethnic identity, historical trauma, and participation in cultural activities: Associations with smoking behaviors among American Indian adolescents in California. Addictive Behaviors, 50, 64-69.

Strauss, A., \& Corbin, J. (1990). Basics of qualitative research: Grounded theory procedures and techniques. Newbury Park, CA: Sage Publications.

Szlemko, W. J., Wood, J. W., \& Thurman, P. J. (2006). Native Americans and alcohol: Past, present, and future. The Journal of General Psychology, 133(4), 435-451. doi: 10.3200/genp.133.4.435-451

U.S. Commission on Civil Rights. (2004). Broken promises: Evaluating the Native American health care system. Washington, D.C.: U.S. Commission on Civil Rights.

Wallerstein, N, \& Duran, B. (2010). Community-based participatory researh contributes to intervention research: The intersection of science and practice to improve health equity. American Journal of Public Health, 100(S1):S40-S46.

Walters, K. L., \& Simoni, J. M. (1999). Trauma, substance use, and HIV risk among urban American Indian women. Cultural Diversity and Ethnic Minority Psychology, 5(3), 236248. doi: 10.1037/1099-9809.5.3.236

Walters, K. L., Simoni, J. M., \& Evans-Campbell, T. (2002). Substance use among American Indians and Alaska Natives: Incorporating culture in an "indigenist" stress-coping paradigm. Public Health Reports, 117(Suppl 1), S104-S117. 
Warren, K. R., Hewitt, B. G., \& Thomas, J. D. (2011). Fetal alcohol spectrum disorders:

Research challenges and opportunities. Alcohol Research \& Health: The Journal of the National Institute on Alcohol Abuse and Alcoholism, 34(1), 4. 